\title{
SYMMETRIES OF ESCHENBURG SPACES AND THE CHERN PROBLEM*
}

\author{
KARSTEN GROVE ${ }^{\dagger}$, KRISHNAN SHANKAR $^{\ddagger}$, AND WOLFGANG ZILLER ${ }^{\S}$ \\ Dedicated to the memory of S. S. Chern.
}

Key words. Positive curvature, Bi-quotients, Isometry groups, Chern Conjecture

AMS subject classifications. Primary 53C20; Secondary 57S15

To advance our basic knowledge of manifolds with positive (sectional) curvature it is essential to search for new examples, and to get a deeper understanding of the known ones. Although any positively curved manifold can be perturbed so as to have trivial isometry group, it is natural to look for, and understand the most symmetric ones, as in the case of homogeneous spaces. In addition to the compact rank one symmetric spaces, the complete list (see [BB]) of simply connected homogeneous manifolds of positive curvature consists of the Berger spaces $B^{7}$ and $B^{13}[\mathrm{Be}]$, the Wallach spaces $W^{6}, W^{12}$ and $W^{24}$ [Wa], and the infinite class of so-called AloffWallach spaces, $\mathcal{A}^{7}[\mathrm{AW}]$. Their full isometry groups were determined in [Sh2], and this knowledge provided new basic information about possible fundamental groups of positively curved manifolds, and in particular to counter-examples of the so-called Chern conjecture (see [Sh1] and [GSh, Ba2]), which states that every abelian subgroup of the fundamental group is cyclic.

Our purpose here is to begin a systematic analysis of the isometry groups of the remaining known manifolds of positive curvature, i.e., of the so-called Eschenburg spaces, $\mathcal{E}^{7}$ [Es1, Es2] (plus one in dimension 6) and the Bazaikin spaces, $\mathcal{B}^{13}$ [Ba1], with an emphasis on the former. In particular, we completely determine the identity component of the isometry group of any positively curved Eschenburg space. A member of $\mathcal{E}$ is a so-called bi-quotient of $\mathrm{SU}(3)$ by a circle:

$$
E=\operatorname{diag}\left(z^{k_{1}}, z^{k_{2}}, z^{k_{3}}\right) \backslash \mathrm{SU}(3) / \operatorname{diag}\left(z^{l_{1}}, z^{l_{2}}, z^{l_{3}}\right)^{-1},|z|=1
$$

with $\sum k_{i}=\sum l_{i}$. Further conditions on the integers are required for $E$ to be a manifold and for the Eschenburg metric to have positive curvature, see (1.1). They contain the homogeneous Aloff-Wallach spaces $\mathcal{A}$, corresponding to $l_{i}=0, i=1,2,3$, as a special subfamily. Similarly, any member of $\mathcal{B}$ is a bi-quotient of $\mathrm{SU}(5)$ by $\mathrm{Sp}(2) \mathrm{S}^{1}$ and the Berger space, $B^{13} \in \mathcal{B}$. It was already noticed several years ago by the first and last author, that both $\mathcal{E}$ and $\mathcal{B}$ contain an infinite family $\mathcal{E}_{1}$ respectively $\mathcal{B}_{1}$ of cohomogeneity one, i.e., they admit an isometric group action with 1-dimensional orbit space (see section 1 and $[\mathrm{Zi}]$ ). There is a larger interesting subclass $\mathcal{E}_{2} \subset \mathcal{E}$, corresponding to $l_{1}=l_{2}=0$, which contains $\mathcal{E}_{1}$ as well as $\mathcal{A}$, and whose members

\footnotetext{
*Received May 1, 2006; accepted for publication July 28, 2006. All three authors were supported by grants from the National Science Foundation and the third author by the Francis J. Carey Term Chair and the Clay Institute.

${ }^{\dagger}$ Department of Mathematics, University of Maryland, College Park, MD 20742, USA (kng@ math.umd.edu).

${ }^{\ddagger}$ Department of Mathematics, University of Oklahoma, Norman, OK 73019, USA (shankar@ math.ou.edu).

$\S$ Department of Mathematics, University of Pennsylvania, Philadelphia, PA 19104, USA (wziller@ math.upenn.edu).
} 
admit an isometric cohomogeneity two action. The remaining spaces $\mathcal{E}-\mathcal{E}_{2}$ all have a cohomogeneity four action. We point out that $\mathcal{E}_{1} \cap \mathcal{A}$ has only one member $A_{1,1}$, the unique Aloff-Wallach space that is also a normal homogeneous space (see [Wi1]).

TheOREM A. The isometry group of any positively curved Eschenburg space $E$ has rank 3 and dimension 11, 9, 7, 5, or 3 corresponding to the cases $E=A_{1,1}$, $E \in \mathcal{A}-\left\{A_{1,1}\right\}, E \in \mathcal{E}_{1}-\left\{A_{1,1}\right\}, E \in \mathcal{E}_{2}-\left(\mathcal{E}_{1} \cup \mathcal{A}\right)$, or $E \in \mathcal{E}-\mathcal{E}_{2}$ respectively.

There are at most two possible groups for each class, and in all cases we know explicitly what they are (at least up to components, see Theorems 2.4, 2.5, 3.6, and 4.1.)

For more information about equivalences up to diffeomorphism, homeomorphism and homotopy within the classes $\mathcal{E}$ and $\mathcal{B}$, we refer to [KS], [Kr2], [Sh3], [CEZ] and [FZ1]. We note in particular that there are spaces from the disjoint classes $\mathcal{E}-\mathcal{E}_{2}$ and $\mathcal{E}_{2}$ that are diffeomorphic, but Theorem A implies they cannot be isometric.

The explicit description of each $E \in \mathcal{E}$ gives rise to a "natural group" of isometries $\mathrm{N}(E) \subset \operatorname{Iso}(E)$ (see section 1) consisting of isometries of the metric on $\mathrm{SU}(3)$ which commute with the circle action. This action has cohomogeneity $0,1,2$ or 4 . In the proof of Theorem A we show that this group cannot be enlarged to a bigger (connected) group of isometries. Here we strongly use the conditions for the Eschenburg space to have positive curvature since it puts severe restrictions on the possible groups that can act isometrically.

Our concrete knowledge of the isometry groups of spaces $E \in \mathcal{E}$ allows us to expand the list of positively curved manifolds with interesting fundamental groups. Here subgroups of $\mathrm{SO}(3)$ are particularly interesting, since many of them do not occur as space form groups, i.e., as fundamental groups of spaces of constant curvature. The non-abelian simple group $A_{5}$ and the abelian non-cyclic group $\mathbb{Z}_{2} \oplus \mathbb{Z}_{2}$ are examples of such groups. We will see that $\mathrm{SO}(3)$ itself acts freely and isometrically on only one Aloff-Wallach space, and one Eschenburg space (already found in [Sh1]). Nevertheless we will show the following, which adds infinitely many spaces with distinct homotopy types that violate Chern's conjecture for fundamental groups of positively curved manifolds.

Theorem B. For any finite subgroup $\Gamma \subseteq \mathrm{SO}(3)$, there exist infinitely many spaces in $\mathcal{E}_{1}$ as well as in $\mathcal{E}_{2}-\mathcal{E}_{1}$ on which $\Gamma$ acts freely and isometrically.

Moreover, for any odd positive integers $p$ and $q$ with $\operatorname{gcd}(p+1, q)=1$ the group $\mathbb{Z}_{2} \times \mathbb{Z}_{2 q}$ acts freely and isometrically on $E_{p} \in \mathcal{E}_{1}$.

We have divided the paper into five sections. In the first section we set up notation, including the precise definitions of the objects we are interested in, and present the tools needed for our proofs. Section 2 deals with the cohomogeneity one spaces $\mathcal{E}_{1}$ and $\mathcal{B}_{1}$. The bulk of our work is in section 3 which provides a detailed analysis of the class $\mathcal{E}_{2}$ (we also include a brief discussion for the 6-dimensional "Eschenburg flag"). Section 4 is concerned with the class $\mathcal{E}-\mathcal{E}_{2}$. In section 5 we use our knowledge of isometry groups developed in sections 2 and 3 to find free isometric actions on manifolds from $\mathcal{E}_{2}$.

It is our pleasure to thank Burkhard Wilking for informing us about how to deal with the spaces in $\mathcal{E}-\mathcal{E}_{2}$ (Theorem 4.1).

1. Preliminaries and tools. The general strategy for determining the isometry groups of the Eschenburg and Bazaikin spaces has two steps. The first and fairly simple step is to exhibit a (connected) group of isometries which arises naturally from the description of the space. In the second and more difficult step we then show 
that it cannot be enlarged to a group of larger dimension. This uses an analysis of orbit spaces and isotropy groups. The possible choices of enlargements are severely restricted by a number of classification theorems about positively curved manifolds with large isometry groups. In this section we will describe the first step, and provide the general tools needed for the second step.

\section{Biquotient metrics and Natural Isometries.}

Throughout the paper, we let $\operatorname{Iso}(M)$ denote the full group of isometries of a Riemannian manifold $M$. As usual, the identity component of a Lie group $\mathrm{G}$ will be denoted by $G_{0}$, and if $H \subset G$ is a closed subgroup, then $N_{G}(H)$ is the normalizer of $\mathrm{H}$ in $\mathrm{G}$, or just $\mathrm{N}(\mathrm{H})$ if it is clear from the context.

By definition, a biquotient manifold $M$ is the orbit space $\mathrm{G} / / \mathrm{U}$ of a compact Lie group $\mathrm{G}$, by a subgroup $\mathrm{U} \subset \mathrm{G} \times \mathrm{G}$ acting freely as

$$
\mathrm{U} \times \mathrm{G} \rightarrow \mathrm{G}, \quad\left(u_{1}, u_{2}\right) \cdot g \rightarrow u_{1} \cdot g \cdot u_{2}^{-1},
$$

When $\mathrm{U}$ lies strictly in one factor of $\mathrm{G} \times \mathrm{G}$, then the quotient is a homogeneous space.

The Riemannian metrics we consider on a biquotient $M=\mathrm{G} / / \mathrm{U}$ are always induced from a left invariant, $\operatorname{Ad}(\mathrm{K})$-invariant metric on $\mathrm{G}$ where $\mathrm{U} \subset \mathrm{G} \times \mathrm{K}$, and $\mathrm{K} \subset \mathrm{G}$ is a closed subgroup. We then have the inclusions $\operatorname{Iso}(M) \supset \mathrm{N}_{\mathrm{Iso}(\mathrm{G})}(\mathrm{U}) / \mathrm{U} \supset$ $\mathrm{N}_{\mathrm{G} \times \mathrm{K}}(\mathrm{U}) / \mathrm{U}$ since $\mathrm{G} \times \mathrm{K} \subset \mathrm{Iso}(\mathrm{G})$. We will refer to $\mathrm{N}(M):=\mathrm{N}_{\mathrm{G} \times \mathrm{K}}(\mathrm{U}) / \mathrm{U}$ as the natural group of isometries of the biquotient $M=\mathrm{G} / / \mathrm{U}$.

\section{Eschenburg and Bazaikin spaces.}

We will now describe the special biquotients we are dealing with in this paper, namely the Eschenburg and the Bazaikin spaces $\mathcal{E}$ and $\mathcal{B}$. For both classes $\mathrm{G}=\mathrm{SU}(n)$ and $\mathrm{K}=\mathrm{U}(n-1)=\mathrm{S}(\mathrm{U}(n-1) \mathrm{U}(1))$, where $n=3$ and 5 respectively. From the above discussion this will already determine the metrics we consider on the orbit spaces $\mathrm{G} / / \mathrm{U}$, for $\mathrm{U} \subset \mathrm{G} \times \mathrm{K}$.

To describe the spaces in $\mathcal{E}^{7}$, we proceed as follows:

Let $\bar{a}:=\left(a_{1}, a_{2}, a_{3}\right), \bar{b}:=\left(b_{1}, b_{2}, b_{3}\right)$ be triples of integers such that $\sum a_{i}=\sum b_{i}:=c$. Let

$$
\mathrm{S}_{\bar{a}, \bar{b}}^{1}=\left\{\left(\operatorname{diag}\left(z^{a_{1}}, z^{a_{2}}, z^{a_{3}}\right), \operatorname{diag}\left(z^{b_{1}}, z^{b_{2}}, z^{b_{3}}\right)\right) \mid z \in \mathrm{U}(1)\right\}
$$

The $\mathrm{S}_{\bar{a}, \bar{b}}^{1}$ action on $\mathrm{SU}(3)$ is free if and only if for every permutation $\sigma \in \mathrm{S}_{3}, \operatorname{gcd}\left(a_{1}-\right.$ $\left.b_{\sigma(1)}, a_{2}-b_{\sigma(2)}\right)=1$. In this case, we will call the resulting 7 -manifold, $E_{\bar{a}, \bar{b}}:=$ $\mathrm{SU}(3) / / \mathrm{S}_{\bar{a}, \bar{b}}^{1}$, an Eschenburg space. Note that $\mathrm{S}_{\bar{a}, \bar{b}}^{1} \subsetneq \mathrm{SU}(3) \times \mathrm{U}(2)$, but its action is the same, up to an ineffective kernel, as that by $\mathrm{S}_{3 \bar{a}-\bar{c}, 3 \bar{b}-\bar{c}}^{1} \subset \mathrm{SU}(3) \times \mathrm{U}(2)$, where $\bar{c}=(c, c, c)$. In [Es1] it was shown that the Eschenburg metric on $E_{\bar{a}, \bar{b}}$ has positive sectional curvature if and only if one of the following holds:

$$
b_{i} \notin\left[a_{\min }, a_{\max }\right], \text { or } a_{i} \notin\left[b_{\min }, b_{\max }\right] \text { for all } i .
$$

Strictly speaking, we need to allow the invariance of the metric to be switched, and to choose any of the 3 different block embeddings of $\mathrm{U}(2) \subset \mathrm{SU}(3)$ in order to obtain this necessary and sufficient condition. But for convenience, we will fix the embedding and assume the metric is left invariant. We reserve the notation $\mathcal{E}$ for those Eschenburg spaces $E_{\bar{a}, \bar{b}}$ that have positive curvature. If the action by $\mathrm{S}_{\bar{a}, \bar{b}}^{1}$ is only one sided, we obtain the subfamily of homogeneous Aloff-Wallach spaces 
$A_{k, l}=\mathrm{SU}(3) / \operatorname{diag}(k, l,-(k+l))$ with $\operatorname{gcd}(k, l)=1$. Here we can assume, up to conjugacy and change of orientation, that $k \geq l \geq 0 . A_{k, l}$ admits a homogeneous metric with positive curvature if and only if $l>0$.

To describe the spaces in $\mathcal{B}$, consider a five tuple of integers $\bar{p}=\left(p_{1}, p_{2}, p_{3}, p_{4}, p_{5}\right)$ with $q:=\sum p_{i}$. Let

$$
\operatorname{Sp}(2) \mathrm{S}_{\bar{p}}^{1}=\left\{\left(\operatorname{diag}\left(z^{p_{1}}, z^{p_{2}}, z^{p_{3}}, z^{p_{4}}, z^{p_{5}}\right), \operatorname{diag}\left(\operatorname{Sp}(2), z^{q}\right)\right\},\right.
$$

where $\mathrm{Sp}(2) \subset \mathrm{SU}(4)$ is embedded in the upper block of $\mathrm{SU}(5)$. The action of $\mathrm{Sp}(2) \mathrm{S}_{\bar{p}}^{1}$ on $\mathrm{SU}(5)$ is free if and only if all $p_{i}$ are odd and for all permutations $\sigma \in \mathrm{S}_{5}, \operatorname{gcd}\left(p_{\sigma(1)}+\right.$ $\left.p_{\sigma(2)}, p_{\sigma(3)}+p_{\sigma(4)}\right)=2$. In this case, we say that $B_{\bar{p}}:=\mathrm{SU}(5) / / \mathrm{Sp}(2) \mathrm{S}_{\bar{p}}^{1}$ is a Bazaikin space. As for the Eschenburg spaces above we note that $\mathrm{Sp}(2) \mathrm{S}_{\bar{p}}^{1} \subsetneq \mathrm{SU}(5) \times \mathrm{U}(4)$, but its action is the same as that of $\mathrm{Sp}(2) \mathrm{S}_{5 \bar{p}-\bar{q}}^{1} \subset \mathrm{SU}(5) \times \mathrm{U}(4)$, where $\bar{q}=(q, q, q, q, q)$. From the treatment in [Zi] of Bazaikin's work [Ba1], we know that the Eschenburg metric on $B_{\bar{p}}$ has positive curvature if and only if

$$
p_{\sigma(1)}+p_{\sigma(2)}>0 \text { for all permutations } \sigma \in \mathrm{S}_{5} .
$$

We reserve the notation $\mathcal{B}$ for those Bazaikin spaces $B_{\bar{p}}$ that have positive curvature. In the case of $\bar{p}=(1, \ldots, 1)$ we obtain the unique Bazaikin space which is homogeneous, the Berger space $B^{13}=\mathrm{SU}(5) / \mathrm{Sp}(2) \mathrm{S}^{1}$.

\section{Group Enlargements.}

In this subsection we consider the situation where an isometric $\mathrm{G}$ action on $M$ is a sub-action of an isometric $\mathrm{G}^{*}$ action, and $\mathrm{G} \subseteq \mathrm{G}^{*}$ and $M$ are all compact and connected. Clearly then, one has an induced submetry $\pi: M / \mathrm{G} \longrightarrow M / \mathrm{G}^{*}$ and $\operatorname{dim}\left(M / \mathrm{G}^{*}\right) \leq \operatorname{dim}(M / \mathrm{G})$. Moreover, if we let $(M / \mathrm{G})_{0}$ denote the regular part of $M / \mathrm{G}$, corresponding to the principal $\mathrm{G}$ orbits $M_{0}$ in $M$, and similarly for the $\mathrm{G}^{*}$ action, we have:

Lemma 1.3 (Submetry). All principal $\mathrm{G}^{*}$ orbits in $M$ are equivalent as $\mathrm{G}$ manifolds as well. Moreover, the subset $(M / \mathrm{G})_{0} \cap \pi^{-1}\left(M / \mathrm{G}^{*}\right)_{0}$ is open and dense in $M / \mathrm{G}$, and the image $\pi\left((M / \mathrm{G})_{0} \cap \pi^{-1}\left(M / \mathrm{G}^{*}\right)_{0}\right)=\left(M / \mathrm{G}^{*}\right)_{0}$. In particular, $M / \mathrm{G}$ and $M / \mathrm{G}^{*}$ are isometric if $\operatorname{dim}(M / \mathrm{G})=\operatorname{dim}\left(M / \mathrm{G}^{*}\right)$.

Proof. Let $P$ and $P^{*}$ denote the projections from $M$ to $M / \mathrm{G}$ and $M / \mathrm{G}^{*}$ respectively. We have the following commutative diagram.

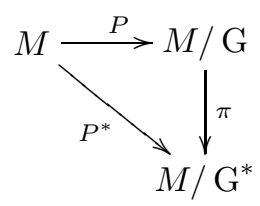

The collection $M_{0}^{*}$ of all principal $\mathrm{G}^{*}$ orbits in $M$ is an open and dense $\mathrm{G}$ invariant subset of $M$. Moreover, all principal $\mathrm{G}^{*}$ orbits are equivalent as $\mathrm{G}$ manifolds as well. Clearly then, the set of principal $\mathrm{G}$ orbits in $M_{0}^{*}$ is open and dense in $M$, and in fact $P^{*}\left(M_{0} \cap M_{0}^{*}\right)=\left(M / \mathrm{G}^{*}\right)_{0}$ and $P\left(M_{0} \cap M_{0}^{*}\right)=(M / \mathrm{G})_{0} \cap \pi^{-1}\left(M / \mathrm{G}^{*}\right)_{0}$.

Now suppose $\operatorname{dim}(M / \mathrm{G})=\operatorname{dim}\left(M / \mathrm{G}^{*}\right)$. Then the map $\pi$ is a local isometry from $(M / \mathrm{G})_{0} \cap \pi^{-1}\left(M / \mathrm{G}^{*}\right)_{0}$ onto $\left(M / \mathrm{G}^{*}\right)_{0}$. Since $\mathrm{G}$ and $\mathrm{G}^{*}$ are connected, it is also clearly 1-1, and hence an isometry. It now follows from the first part that $M / \mathrm{G}$ and $M / \mathrm{G}^{*}$ are isometric under $\pi$. 
Size Restrictions.

In the presence of positive curvature, the size of the isometry group is restricted, which will be an important tool in our discussions.

Recall that the symmetry rank of a manifold by definition is the rank of its isometry group. In positive curvature this rank is bounded above by [GS1].

THEOREM 1.4 (Rank Rigidity). Assume that a $k$-dimensional torus acts effectively and isometrically on a positively curved simply connected $n$-manifold $M$. Then $k \leq\left[\frac{n+1}{2}\right]$, and equality holds only when $M$ is diffeomorphic to $\mathbb{S}^{n}$ or $\mathbb{C P}^{n / 2}$.

Another measurement for the size of a group is its dimension. The degree of symmetry of a Riemannian manifold $M$ is by definition the dimension of its isometry group. This dimension is severely restricted in positive curvature by the following result of Wilking [Wi3]

ThEOREM 1.5 (Symmetry Degree). Let $\left(M^{n}, g\right)$ be a simply connected, Riemannian manifold of positive curvature. If the symmetry degree of $M^{n}$ is at least $2 n-6$, then $M^{n}$ is homotopy equivalent to a compact, rank one symmetric space, or $M^{n}$ is isometric to a homogeneous space of positive sectional curvature.

The cohomogeneity, i.e., the dimension of the orbit space gives yet another measurement for the size of a transformation group. A related invariant is the so-called fixed point cohomogeneity which is the dimension of the normal sphere to the fixed point set in the orbit space. A manifold that supports an action of fixed point cohomogeneity 0 is called fixed point homogeneous. Although this will not be used in the sense of size here, the following classification results of [GS2] and [GK] are quite useful for our investigations:

THEOREM 1.6 (Low cofix). A simply connected manifold of positive curvature with fixed point cohomogeneity at most one, is diffeomorphic to a rank one symmetric space.

2. Cohomogeneity One. In this section we single out the subclasses $\mathcal{E}_{1} \subset \mathcal{E}$ and $\mathcal{B}_{1} \subset \mathcal{B}$ of positively curved cohomogeneity one Eschenburg and Bazaikin spaces, and determine their full isometry groups. Here

$$
\mathcal{E}_{1}=\left\{E_{p}=E_{\bar{a}, \bar{b}} \in \mathcal{E} \mid \bar{a}=(1,1, p), \bar{b}=(0,0, p+2), p>0\right\}
$$

and

$$
\mathcal{B}_{1}=\left\{B_{p}=B_{\bar{p}} \in \mathcal{B} \mid \bar{p}=(1,1,1,1,2 p-1), p>0\right\}
$$

From (1.1) and (1.2) we know that all these manifolds have positive curvature when equipped with the Eschenburg biquotient metric. We also point out that the $\mathrm{S}_{p}^{1}$, and $\mathrm{S}_{-p-1}^{1}$ actions on $\mathrm{SU}(3)$ are equivalent via the inverse map of $\mathrm{SU}(3)$. Moreover, $E_{0} \approx E_{-1}$ only has non-negative curvature in the Eschenburg metric, and in fact does not support any cohomogeneity one metric of positive curvature by [GWZ]. Note also, that $E_{1}$ is the homogeneous Aloff-Wallach space $A_{1,1}$ and $B_{1}$ is the homogeneous Berger space $B^{13}$.

To see that each $E_{p}$ has cohomogeneity one note that the natural action by $\mathrm{U}(2) \times \mathrm{SU}(2)$ (as well as by $\mathrm{SU}(2) \times \mathrm{U}(2)$ ) on $\mathrm{SU}(3)$ commutes with the $\mathrm{S}_{p}^{1}$ action, and that $\mathrm{U}(2) \backslash \mathrm{SU}(3) / \mathrm{SU}(2)=\mathbb{C P}^{2} / \mathrm{SU}(2)$, which is an interval. We also note that $\mathrm{SU}(2) \times \mathrm{SU}(2)$ and $\mathrm{S}_{p}^{1}$ generates $\mathrm{U}(2) \times \mathrm{SU}(2)$ and hence the sub-action by $\mathrm{SU}(2) \times$ $\mathrm{SU}(2)$ is cohomogeneity one as well. 
In the case of $B_{p}$ we see that the natural left action by U(4) on SU(5) commutes with the action of $\operatorname{Sp}(2) \mathrm{S}_{p}^{1}$ which in turn has cohomogeneity one. Indeed, the induced action by $\mathrm{Sp}(2)$ on $\mathrm{U}(4) \backslash \mathrm{SU}(5)=\mathbb{C P}^{4}$ is the standard sub-action of $\mathrm{Sp}(2) \subset \mathrm{SU}(4) \subset$ $\mathrm{SU}(5)$ and hence, $\mathrm{U}(4) \backslash \mathrm{SU}(5) / \mathrm{Sp}(2)$ is an interval. As in the case of Eschenburg spaces, we note that the group generated by $\mathrm{SU}(4)$ and $\operatorname{Sp}(2) \mathrm{S}_{p}^{1}$ is the same as the one generated by $\mathrm{U}(4)$ and $\mathrm{Sp}(2)$, and therefore the sub-action by $\mathrm{SU}(4)$ is also of cohomogeneity one.

Using the tools from section 1 one can prove that if any of these actions extend to a transitive isometric action then $p=1$ for both classes. It is actually known that none of $E_{p}$ or $B_{p}$ for $p>1$ is even homeomorphic to a homogeneous space (see [Sh3], [FZ1]). Since the full isometry groups of these homogeneous spaces were determined in [Sh1] it remains to consider $E_{p}$ and $B_{p}$ for $p>1$.

We first determine the identity component of $\operatorname{Iso}\left(E_{p}\right)$ by analyzing the sub-action by $\mathrm{G}=\mathrm{SU}(2) \times \mathrm{SU}(2) \subset \mathrm{U}(2) \times \mathrm{SU}(2)$, which we noted above is also cohomogeneity one. It is important for us, however, to determine the associated group diagram, $\mathrm{H} \subset\left\{\mathrm{K}^{-}, \mathrm{K}^{+}\right\} \subset \mathrm{G}$, i.e., the isotropy groups along a minimal geodesic between the two non-principal orbits $B_{ \pm}=\mathrm{G} / \mathrm{K}^{ \pm}$corresponding to the end points of the orbit space interval. This information is also used as a recognition tool in the classification work of [GWZ].

Proposition 2.3. The cohomogeneity one action of $\mathrm{G}=\mathrm{SU}(2) \times \mathrm{SU}(2)$ on $E_{p}$ has principal isotropy group $\mathrm{H}=\left\{( \pm \mathrm{id})^{p+1},( \pm \mathrm{id})^{p}\right\} \cong \mathbb{Z}_{2}$ and singular isotropy groups $\mathrm{K}^{-}=\Delta \mathrm{SU}(2) \cdot \mathrm{H}$ and $\mathrm{K}^{+}=\mathrm{S}_{(p+1, p)}^{1}$ embedded with slope $(p+1, p)$ in a maximal torus of $\mathrm{SU}(2) \times \mathrm{SU}(2)$.

Proof. Consider the point $p_{-}=\mathrm{S}_{p}^{1}(e)=\left\{\operatorname{diag}\left(z, z, \bar{z}^{2}\right)\right\}$ in $E_{p}$, and let $B_{-}=$ $\mathrm{G}\left(p_{-}\right)$be the orbit of this point under the action of $\mathrm{SU}(2) \times \mathrm{SU}(2)$. The identity component of the isotropy group at $p_{-}$is clearly $\mathrm{K}_{0}^{-}=\Delta \mathrm{SU}(2)$ and the second component in $\mathrm{K}$ is generated by (id, - id). Hence $B_{-} \cong \mathrm{SO}(3) \cong \mathbb{R}^{3}$.

Since we already saw that $E_{p}$ is cohomogeneity one, the action of $\mathrm{K}^{-}$(effectively by $\left.\mathrm{K}_{0}^{-}=\mathrm{SU}(2)\right)$ on the normal space $T_{p_{-}}^{\perp}$ to $B_{-}$at $p_{-}$is the standard action of $\mathrm{SU}(2)$ on $\mathbb{C}^{2} \cong \mathbb{R}^{4}$. Because this action of $\mathrm{SU}(2)$ restricted to the normal 3-sphere is both transitive and free, the effective version of the action by $\mathrm{G}$ has trivial principal isotropy group.

To find the other singular orbit $B_{+}$, note that $v_{-}=E_{13} \in \mathfrak{s u}(3)$ (standard basis element for the skew symmetric matrices) represents a normal vector to $B_{-}$at $p_{-}$. One easily checks that the one parameter group $\exp \left(t v_{-}\right)$is still a geodesic in the left invariant Eschenburg metric on $\mathrm{SU}(3)$ and hence on $E_{p}$ as well. It intersects $B_{-}$ again at time $\pi$, and not earlier. This implies that $p_{+}=\exp \left(\frac{\pi}{2} v_{-}\right) \in B_{+}$, and $p_{+}$is represented by $E_{13}+\operatorname{diag}(0,1,0)$. To determine $\mathrm{K}^{+}$, let $\left(g_{1}, g_{2}\right) \in \mathrm{SU}(2) \times \mathrm{SU}(2) \subset$ $\mathrm{SU}(3) \times \mathrm{SU}(3)$. We identify $\mathrm{SU}(2)$ with the unit sphere in $\mathbb{C}^{3}$ as usual and let $(a, b)$ correspond to $g_{1}$ and $(\alpha, \beta)$ to $g_{2}$. Then $\left(g_{1}, g_{2}\right) \in \mathrm{K}^{+}$if and only if $\left(g_{1}, g_{2}\right) \cdot p_{+} \epsilon$ $\mathrm{S}_{p}^{1}\left(p_{+}\right)$. This implies that $b=\beta=0, a=\bar{z}^{p+1}$ and $\alpha=\bar{z}^{p}$. Or equivalently, $a=z^{p+1}$ and $\alpha=z^{p}$. Notice that if $p$ is even, then $\mathrm{S}_{(p+1, p)}^{1}$ goes through (-id,id) while it goes through (id, - id) if $p$ is odd and hence $\mathrm{H}=\left\{( \pm \mathrm{id})^{p+1},( \pm \mathrm{id})^{p}\right\}$.

The action of $\mathrm{G}$ is ineffective with kernel $\mathrm{H}$, and hence the natural group of isometries is $\mathrm{U}(2) \times \mathrm{SO}(3)$ when $p$ is odd, and $\mathrm{SO}(3) \times \mathrm{U}(2)$ when $p$ is even. Furthermore, complex conjugation on $\mathrm{SU}(3)$ normalizes the circle action and hence induces an isometry as well. 
THEOREM 2.4. For any integer $p>1$, the full isometry group of $E_{p}$ is given by

$$
\operatorname{Iso}\left(E_{p}\right)=\left(\mathrm{U}(2) \rtimes \mathbb{Z}_{2}\right) \times \mathrm{SO}(3),
$$

where the second component is induced by complex conjugation in $\mathrm{SU}(3)$.

Proof. We already saw that $\mathrm{U}(2) \times \mathrm{SO}(3) \subset \operatorname{Iso}_{0}\left(E_{p}\right)$. Since $E_{p}, p>1$ is not diffeomorphic to a homogeneous space ([Sh3]), any extension of the group action must again be of cohomogeneity one. Moreover, since the two singular orbits $B_{-}$ and $B_{+}$are non-isometric (not even of the same dimension), it must act trivially on the orbit space interval. In other words any extension of the action will have the same orbits. We now consider the codimension two orbit $B_{+}=\mathrm{S}^{3} \times \mathrm{S}^{3} / \mathrm{S}_{(p+1, p)}^{1}$ and claim that the action of $\operatorname{Iso}\left(E_{p}\right)$ on $B_{+}$must be effective. Indeed, assume that an element $\gamma \in \operatorname{Iso}\left(E_{p}\right)$ acts trivially on $B_{+}$. Then $B_{+}$is either totally geodesic, or $\gamma$ acts by reflection on the two dimensional normal space to $B_{+}$. The former is impossible since $B_{+}$does not support a homogeneous metric of positive curvature. The latter is impossible as well, since $E_{p}$ would then have a totally geodesic hypersurface, but in positive curvature this is only possible when the manifold is homeomorphic to the sphere or to real projective space.

We will now examine the size of the isometry group for a homogeneous metric on $B_{+}$. We already know that $\mathrm{G}=\mathrm{S}^{3} \times \mathrm{S}^{3} \times \mathrm{S}^{1}$ (effectively $\mathrm{U}(2) \times \mathrm{SO}(3)$ ) acts by isometries on $B_{+}$. From the classification of 5 -dimensional homogeneous manifolds it follows that it is also the identity component of the isometry group, i.e., no larger connected group can act transitively. One easily checks that $\mathrm{L}=$ $\left\{\operatorname{diag}\left(z^{p+1}, z^{p}, 1\right), \operatorname{diag}(1, w, w)\right\}$ is the full isotropy group of $\mathrm{S}^{3} \times \mathrm{S}^{3} \times \mathrm{S}^{1}$ and that $\mathrm{N}(\mathrm{L}) /(\mathrm{L} \cdot Z(\mathrm{G}))$ is trivial. Furthermore, there exists an outer automorphism of $S^{3} \times S^{3} \times S^{1}$, unique up to inner automorphisms, which preserves L. From [WZ, Theorem 3.1], (cf. also [Sh2]), it then follows that the isometry group of any homogeneous metric on $B_{+}$can have at most two components. Altogether, this completes the proof.

$\mathrm{c}$

We now turn to the isometry groups of the Bazaikin spaces $B_{p} \in \mathcal{B}_{1}, p>1$. We already saw that the natural left $\mathrm{U}(4)$ action on $\mathrm{SU}(5)$ induces a cohomogeneity one action on $B_{p}$. Furthermore, complex conjugation in $\mathrm{SU}(5)$ generates a second component.

THEOREM 2.5. The full isometry group of the cohomogeneity one manifold $B_{p}, p>1$ may be written as:

$$
\operatorname{Iso}\left(B_{p}\right)=\mathrm{U}(4) \rtimes \mathbb{Z}_{2},
$$

where the second component is induced by complex conjugation in $\mathrm{SU}(5)$.

Proof. We proceed as in the case of Eschenburg spaces above. Since $B_{p}, p>1$ is not diffeomorphic to a homogeneous space ([FZ1]), any extension of the group action will have cohomogeneity one and in fact the same orbits when the singular orbits are different.

To determine the orbit structure of the action (cf. also [GWZ]) we consider the orbit equivalent sub-action by $\mathrm{G}=\mathrm{SU}(4) \subset \mathrm{U}(4)$. The orbit through the identity is $\mathrm{SU}(4) /(\mathrm{Sp}(2) \cup i \mathrm{Sp}(2))=\mathbb{R} \mathbb{P}^{5}$ and the action by $\mathrm{K}^{-}=\mathrm{Sp}(2) \cup i \mathrm{Sp}(2)$ on the slice is nontrivial and hence $\mathrm{H}=\mathrm{SU}(2) \cdot \mathbb{Z}_{2}$. Since $B_{p}$ is simply connected, the cohomogeneity one action cannot have any exceptional orbits and since $\mathrm{K}^{+} / \mathrm{H}$ is a sphere and $\mathrm{H}$ is not connected, it must be one dimensional, i.e., $\mathrm{K}^{+}=\mathrm{SU}(2) \cdot \mathrm{S}^{1}$. Since the centralizer of $\mathrm{H}$ 
in $\mathrm{G}$ is two dimensional, $\mathrm{S}^{1}$ is allowed to have slopes $(r, s)$ inside this two-torus. These slopes are then determined from those for the Eschenburg spaces,since $E_{p}$ is the fixed point set of the involution $\operatorname{diag}(1,1,-1,-1,1) \in \mathrm{SU}(5)$, assuming that $\mathrm{SU}(2) \subset \mathrm{H}$ is the lower $2 \times 2$ block of $\mathrm{SU}(5)$, (see [Ta]). It thus follows that $(r, s)=(p+1, p)$. Note that since $E_{p}$ is not homogeneous, this observation also provides a simple geometric proof that $B_{p}$ cannot be homogeneous, since a totally geodesic submanifold of a homogeneous space is itself homogeneous (cf. [KN], Chapter VII, Corollary 8.10).

We again have that $\operatorname{Iso}\left(B_{p}\right)$ acts effectively on $B_{+}=\mathrm{G} / \mathrm{K}^{+}$since $B_{+}$does not support a homogeneous metric with positive curvature, and next determine the isometry group of the metric on $B_{+}$. To see that $\mathrm{U}(4)$ is the identity component, one uses [On, Theorem 4.1] to show that $\mathrm{U}(4)$ cannot be enlarged to a bigger transitive action. By computing the isotropy representation of $\mathrm{G} / \mathrm{K}^{+}$, it follows that $\mathrm{N}\left(\mathrm{K}^{+}\right) \subset \mathrm{SU}(2) \cdot \mathrm{U}(2)$ and hence $\mathrm{N}\left(\mathrm{K}^{+}\right) / \mathrm{K}^{+}=\mathrm{S}^{1}$ is connected. Since furthermore, $\mathrm{U}(4)$ has, up to inner automorphisms, a unique outer automorphism, [WZ, Theorem 3.1] finishes the proof.

3. Cohomogeneity Two. In this section we define and analyze a subclass $\mathcal{E}_{2} \subset$ $\mathcal{E}$ of positively curved, cohomogeneity two Eschenburg spaces, and determine their isometry groups. We will also briefly treat the single 6-dimensional Eschenburg space, $E^{6}$.

Define

$$
\mathcal{E}_{2}=\left\{E_{\bar{p}}=E_{\bar{a}, \bar{b}} \in \mathcal{E} \mid \bar{a}=\left(p_{1}, p_{2}, p_{3}\right), \bar{b}=\left(0,0, p_{1}+p_{2}+p_{3}\right)\right\}
$$

We note that the $\mathrm{S}_{\bar{p}}^{1}$ action on $\mathrm{SU}(3)$ is free if and only if $\operatorname{gcd}\left(p_{i}, p_{j}\right)=1$ for all $i \neq j$. In particular at most one $p_{i}$ is even. From (1.1) it easily follows that the Eschenburg metric has positive curvature, if and only if, up to reordering of the $p_{i}$ 's and changing the sign of all three, one of the following holds:

$$
0<p_{1} \leq p_{2} \leq p_{3} \text { or } 0<p_{2} \leq p_{3} \text { and } p_{1}<-p_{3}
$$

This class obviously contains the cohomogeneity one Eschenburg spaces, $E_{p} \in \mathcal{B}_{1}$, where $\bar{p}=(1,1, p)$. Moreover, it contains the (homogeneous) Aloff-Wallach spaces $\mathcal{A}$, since $A_{p_{1}, p_{2}}=E_{\bar{p}}$ when $p_{3}=-\left(p_{1}+p_{2}\right)$.

Note that the natural action on $\mathrm{SU}(3)$ by $\mathrm{T}^{2} \times \mathrm{U}(2)$ commutes with the $\mathrm{S}_{\bar{p}}^{1}$ action, and that $T^{2} \backslash \mathrm{SU}(3) / \mathrm{U}(2)=\mathrm{T}^{2} \backslash \mathbb{C} \mathbb{P}^{2}$ is a right angled triangle. Since $\mathrm{S}_{\bar{p}}^{1}$ and $\mathrm{T}^{2} \times \mathrm{SU}(2)$ generate $\mathrm{T}^{2} \times \mathrm{U}(2)$, we see that the induced action by $\mathrm{T}^{2} \times \mathrm{SU}(2)$ on $E_{\bar{p}}$ has cohomogeneity two. Clearly the effective group is $\mathrm{T}^{2} \times \mathrm{SO}(3)$ when all $p_{i}$ are odd, and $\mathrm{T}^{2} \times \mathrm{SU}(2)$ otherwise. For convenience we will work with $\mathrm{T}^{2} \times \mathrm{SU}(2)$ directly. Although we do not need the full orbit structure of this cohomogeneity two action, the following information will be crucial.

Lemma 3.3. The orbits of the $\mathrm{T}^{2} \times \mathrm{SU}(2)$ action on $E_{\bar{p}}$, corresponding to the vertices in the quotient triangle, are lens spaces with fundamental groups of order $\left|p_{i}+p_{j}\right|$.

Proof. Let us first consider the orbit $B_{1}$ going through the image of id $\in \mathrm{SU}(3)$ in $E_{\bar{p}}$. The element $\left(\left(w_{1}, w_{2}\right), \operatorname{diag}(r, \bar{r})\right) \in \mathrm{T}^{2} \times \mathrm{SU}(2)$ lies in the isotropy of this point if and only if $\operatorname{diag}\left(w_{1} r, w_{2} \bar{r}, \bar{w}_{1} \bar{w}_{2}\right)=\operatorname{diag}\left(z^{p_{1}}, z^{p_{2}}, \bar{z}^{p_{1}+p_{2}}\right)$ for some $z \in \mathrm{U}(1)$. Since $w_{1}$ and $w_{2}$ can be described arbitrarily, the subgroup $1 \times \mathrm{SU}(2)$ acts transitively on $B_{1}$ and has isotropy group $\operatorname{diag}\left(z^{p_{1}}, z^{p_{2}}\right)$ with $z^{p_{1}+p_{2}}=1$, i.e., $B_{1}$ is a lens space with fundamental group $\mathbb{Z}_{\left|p_{1}+p_{2}\right|}$. 
The two other vertices can be represented by $E_{13}+\operatorname{diag}(0,1,0)$ and $E_{23}+$ $\operatorname{diag}(1,0,0)$ since they are the endpoints of a geodesic of length $\pi / 2$ with initial vectors orthogonal to $B_{1}$ and singular with respect to the isotropy action on the normal space of $B_{1}$. The same computation as above shows that the orbits through these two points are lens spaces with fundamental group $\mathbb{Z}_{\left|p_{1}+p_{3}\right|}$ and $\mathbb{Z}_{\left|p_{2}+p_{3}\right|}$ respectively.

In order to determine the full isometry group, we need to prove the following claims:

- If the $\mathrm{T}^{2} \times \mathrm{SU}(2)$ action on $E_{\bar{p}} \in \mathcal{E}_{2}$ extends to an isometric cohomogeneity one action, then $p_{1}=p_{2}=0$, i.e., $E_{\bar{p}} \in \mathcal{E}_{1}$.

- If the $\mathrm{T}^{2} \times \mathrm{SU}(2)$ action on $E_{\bar{p}} \in \mathcal{E}_{2}$ extends to a transitive isometric action, then $p_{3}=-p_{1}-p_{2}$, i.e., $E_{\bar{p}} \in \mathcal{A}$.

We point out that classification results for positively curved manifolds in cohomogeneity zero and one immediately yield diffeomorphism conclusions in the above cases, but our results are about equality of the integer parameters.

Recall that by the rank rigidity theorem (1.4), Iso $\left(E_{\bar{p}}\right)$ must have rank 3 , and by the degree theorem $(1.5), \operatorname{dim} \operatorname{Iso}\left(E_{\bar{p}}\right) \leq 8$, unless $E_{\bar{p}}$ is isometric to a homogeneous space with positive curvature. This leaves only the following possible (almost effective) connected extensions $\mathrm{G}^{*}$ of $\mathrm{G}=\mathrm{T}^{2} \times \mathrm{SU}(2)$ :

- $\mathrm{G}^{*}=\mathrm{S}^{1} \times \mathrm{SU}(2) \times \mathrm{SU}(2)$

- $\mathrm{G}^{*}=\mathrm{S}^{1} \times \mathrm{SU}(3)$, or $\mathrm{G}^{*}=\mathrm{SU}(2) \times \mathrm{SU}(3)$

We first deal with the latter extension case:

Proposition 3.4 (Homogeneous Case). Let $E_{\bar{p}} \in \mathcal{E}_{2}$, and assume the action of $\mathrm{G}=\mathrm{T}^{2} \times \mathrm{SU}(2)$ extends to a larger (connected) group $\mathrm{G}^{*}$ with $\operatorname{dim}\left(\mathrm{G}^{*}\right)>8$, then one of the following must occur:

(i) $\mathrm{G}^{*}=\mathrm{SU}(2) \times \mathrm{SU}(3), \bar{p}=(1,1,-2)$ and $E_{\bar{p}}$ is the Aloff-Wallach space $A_{1,1}$.

(ii) $\mathrm{G}^{*}=\mathrm{S}^{1} \times \mathrm{SU}(3), \bar{p}=\left(p_{1}, p_{2},-p_{1}-p_{2}\right)$ and $E_{\bar{p}}$ is the Aloff-Wallach space $A_{p_{1}, p_{2}}$.

Proof. The degree theorem implies that under the assumption $\operatorname{dim}\left(G^{*}\right)>8$, the Eschenburg space must be isometric to a homogeneous space. Since among the positively curved homogeneous spaces in dimension 7, only the Aloff-Wallach spaces have possibly the same homotopy type as an Eschenburg space ([Es1]), it only remains to check the claims about $\bar{p}$.

In the first case we note that only $A_{1,1}$ has an 11-dimensional isometry group (cf. [Sh2]). To see that indeed $\bar{p}=(1,1,-2)$, we use the fact that the fourth cohomology group of $E_{\bar{p}}$ is a finite cyclic group of order $r=\left|p_{1} p_{2}+p_{1} p_{3}+p_{2} p_{3}\right|$ (cf. [Es3]). Using the positive curvature condition (3.2), one easily sees that $r=3$ is only assumed in the case of $\bar{p}=(1,1,-2)$.

In the second case, observe that there is, up to conjugacy, only one immersed subgroup $\mathrm{T}^{2} \times \mathrm{SU}(2)$ in $\mathrm{S}^{1} \times \mathrm{SU}(3)$. Thus the cohomogeneity two action agrees with the one considered above on the Aloff-Wallach spaces $A_{k, l}$ and hence the vertices in the orbit space correspond to 3-dimensional lens spaces with fundamental groups of orders $|k|,|l|,|k+l|$. On the other hand, for the action of $\mathrm{T}^{2} \times \mathrm{SU}(2)$ on $E_{\bar{p}}$ the vertices correspond to lens spaces whose fundamental groups have orders $\left|p_{i}+p_{j}\right|$. This imposes severe restrictions on the $p_{i}$ 's, and one easily shows that under the positive curvature condition (3.2) this is only possible when $\sum p_{i}=0$. $\square$

For the first extension case, we will show:

Proposition 3.5 (Cohomogeneity One Case). Let $E_{\bar{p}} \in \mathcal{E}_{2}$, and assume that the (almost effective) action of $\mathrm{G}=\mathrm{T}^{2} \times \mathrm{SU}(2)$ extends to a larger (connected) group $\mathrm{G}^{*}$ with $\operatorname{dim}\left(\mathrm{G}^{*}\right) \leq 7$. Then $\mathrm{G}^{*}=\mathrm{S}^{1} \times \mathrm{SU}(2) \times \mathrm{SU}(2)$ and $\bar{p}=(1,1, p), p>0$. 
Proof. We have already seen that $\mathrm{G}^{*}=\mathrm{S}^{1} \times \mathrm{SU}(2) \times \mathrm{SU}(2)$. It remains to show that the action by $\mathrm{G}^{*}$ must be of cohomogeneity one, and then to recognize $\bar{p}$.

If the $\mathrm{G}^{*}$ action also has cohomogeneity two, we see from the submetry lemma (1.3), that it is orbit equivalent to the $\mathrm{G}$ action. Since the latter must have finite principal isotropy group, the fundamental group of the common principal orbits have $\mathbb{Z}$-rank at least 2 . On the other hand, any homogeneous quotient of $G^{*}$ has fundamental group with $\mathbb{Z}$-rank at most 1 , and we conclude that the $\mathrm{G}^{*}$ cation must have cohomogeneity one.

Now consider the submetry $\pi: M / \mathrm{G} \rightarrow M / \mathrm{G}^{*}$. From the submetry lemma, we see that the inverse image of one of the end points of the interval $M / \mathrm{G}^{*}$ is a vertex of the right angled triangle $M / \mathrm{G}$, and that the opposite side is the inverse image of the other end point. Let us denote the common singular $\mathrm{G}$ and $\mathrm{G}^{*}$ orbit by $B_{1}=B_{-}^{*}$, and the other singular $\mathrm{G}^{*}$ orbit by $B_{+}^{*}$. Then $B_{+}^{*}$ is a cohomogeneity one $\mathrm{G}$ manifold at maximal distance to $B_{1}$.

As we saw, $B_{1}$ is a lens space with fundamental group of order $\left|p_{i}+p_{j}\right|$ for some $i, j$ and now the bigger group $\mathrm{G}^{*}=\mathrm{S}^{1} \times \mathrm{SU}(2) \times \mathrm{SU}(2)$ also acts transitively on $B_{1}$. This implies that $B_{1}$ is either $\mathbb{S}^{3}$ or $\mathbb{R} \mathbb{P}^{3}$, i.e., that $\left|p_{i}+p_{j}\right| \leq 2$. Indeed, if not, the $\mathrm{G}^{*}$ action on $B_{1}$ has a kernel $C$ of dimension at least 3 since the isometry group of any lens space $\mathbb{S}^{3} / \mathbb{Z}_{m}$, with $m \geq 3$ has dimension at most 4 . In particular, $C$ must contain an $\mathrm{SU}(2) \subset \mathrm{G}^{*}$. Since $B_{1}$ has codimension 4 , this $\mathrm{SU}(2)$ acts either trivially or transitively on the normal spheres to $B_{1}$. In the first case $\mathrm{SU}(2)$ then acts trivially on $M$, and in the second case $M$ is fixed point homogeneous. Either one yields a contradiction (the second by 1.6).

Our next claim is that the two other vertex $\mathrm{G}$ orbits, $B_{2}$ and $B_{3}$, are lens spaces with isomorphic fundamental groups. To see this, consider the cohomogeneity one $G$ manifold $B_{+}^{*}=\mathrm{G}^{*} / \mathrm{K}^{+*}$. The singular orbits $B_{-}=B_{2}$ and $B_{+}=B_{3}$ are again 3dimensional lens spaces with isotropy group $\mathrm{T}^{2}$, and the principal orbits have isotropy $\mathrm{S}^{1}$. In particular $\operatorname{dim} B_{+}^{*}=5$, and $\operatorname{since} \operatorname{codim} B_{-}^{*}=4>2$ it follows that $B_{+}^{*}$ is simply connected by transversality. Thus $\mathrm{K}^{+*}=\mathrm{T}^{2}$ and $B_{+}^{*}=\mathrm{S}^{1} \times \mathrm{SU}(2) \times \mathrm{SU}(2) / \mathrm{T}^{2}=$ $\mathrm{SU}(2) \times \mathrm{SU}(2) / \mathrm{S}_{s, t}^{1}$ for some $s, t$. Note also, that since the $\mathrm{SU}(2) \subset \mathrm{G}$ commutes with $\mathrm{T}^{2}$ it must be one of the $\mathrm{SU}(2)$ factors of $\mathrm{G}^{*}$, and we have a sub-action by $\mathrm{G}^{\prime}=\mathrm{S}^{1} \times \mathrm{SU}(2) \subset \mathrm{SU}(2) \times \mathrm{SU}(2)$ which is orbit equivalent to the $\mathrm{G}$ action on $B_{+}^{*}$. The isotropy groups of the $\mathrm{G}^{\prime}$ action are the intersections of $\mathrm{S}^{1} \times \mathrm{SU}(2)$ with all conjugates of $\mathrm{S}_{s, t}^{1}$. Since obviously both singular isotropy groups are 1-dimensional they are $\mathrm{S}_{s, t}^{1}$ and $\mathrm{S}_{-s, t}^{1}$, and thus both $B_{ \pm}$have fundamental group $\mathbb{Z}_{|s|}$.

We now combine the information $\left|p_{i}+p_{j}\right| \leq 2$ and $\left|p_{i}+p_{k}\right|=\left|p_{k}+p_{j}\right|$ gained so far, with the conditions for positive curvature. One easily sees that this is only possible if either $\bar{p}=(1,1, p)$ with $p>0$ or $\bar{p}=(-k, 1, k-2)$ with $k$ odd and $k \geq 5$. To exclude the latter case we use the fact that by [GWZ] the only manifolds where $\mathrm{S}^{1} \times \mathrm{SU}(2) \times \mathrm{SU}(2)$ acts by cohomogeneity one are the Eschenburg spaces $E_{p}$ for some $p$. We will now see that this contradicts topological invariants for these spaces. Any Eschenburg space has finite, cyclic fourth cohomology group, and for $E_{\bar{p}} \in \mathcal{E}_{2}$, this order is $r=\left|p_{1} p_{2}+p_{1} p_{3}+p_{2} p_{3}\right|$. Kruggel also computed the first Pontrjagin class of an Eschenburg space [Kr1] and it follows in particular that $p_{1}\left(E_{\bar{p}}\right) \equiv 2\left(p_{1}+p_{2}+p_{3}\right)^{2}$ $\bmod r$. The cohomogeneity one manifold $E_{p}$ therefore has $r=2 p+1$ and $p_{1} \equiv p+5$ $\bmod r$ whereas $E_{(-k, 1, k-2)}$ satisfies $r=(k-1)^{2}+1$ and $p_{1} \equiv 2 \bmod r$. This yields the desired contradiction. $\mathrm{Q}$

In the remaining cases it follows that: 
Theorem 3.6 (Cohomogeneity two Case). The identity component of the isometry group of an $E_{\bar{p}} \in \mathcal{E}_{2}-\left(\mathcal{E}_{1} \cup \mathcal{A}\right)$ is given by

$$
\operatorname{Iso}_{0}\left(E_{\bar{p}}\right)=\mathrm{T}^{2} \times \mathrm{S}
$$

where $\mathrm{S}=\mathrm{SO}(3)$ when all $p_{i}$ are odd, and $\mathrm{S}=\mathrm{SU}(2)$ otherwise.

As in the previous cases, there exists another component of the isometry group generated by complex conjugation in $\mathrm{SU}(3)$. We suspect that this is then the full isometry group, but were not able to prove it.

We end this section with a brief discussion of the isometry group of Eschenburg's so-called twisted flag. This is the $\mathrm{T}^{2}$ biquotient of $\mathrm{SU}(3)$ defined by

$$
E^{6}:=\mathrm{SU}(3) / / \mathrm{T}^{2}, \text { where } \mathrm{T}^{2}=\left\{\left(\operatorname{diag}(z, w, z w), \operatorname{diag}\left(1,1, z^{2} w^{2}\right)\right) \mid z, w \in \mathrm{U}(1)\right\} .
$$

The metric on $E^{6}$ induced from the $\mathrm{AdU}(2)$ invariant metric on $\mathrm{SU}(3)$ used in the previous sections has positive curvature (cf. [Es2]). The right action of $\mathrm{U}(2)=$ $\mathrm{S}(\mathrm{U}(2) \mathrm{U}(1)) \subset \mathrm{SU}(3)$ commutes with the $\mathrm{T}^{2}$ action and in particular induces an isometric action on $E$. Moreover, as before, $E / \mathrm{U}(2)=\mathrm{T}^{2} \backslash \mathrm{SU}(3) / \mathrm{U}(2)=\mathrm{T}^{2} \backslash \mathbb{C P}^{2}$ is a triangle and thus $E$ has cohomogeneity two. There are in fact no more isometries in the identity component of the isometry group.

Proposition 3.7. The identity component $\operatorname{Iso}_{0}\left(E^{6}\right)$ of the twisted flag $E^{6}$ is $\mathrm{U}(2)$.

Proof. In [Sh3] it was shown that $E$ is not homotopy equivalent to any homogeneous space and in [Se] that $E$ does not support any positively curved cohomogeneity one metric. So any potential extension of the $\mathrm{U}(2)$ action is again by cohomogeneity two.

From the rank rigidity theorem (1.4) we know that Iso $\left(E^{6}\right)$ has rank at most 2, and from the degree theorem (1.5) that $\operatorname{dim} \operatorname{Iso}\left(E^{6}\right) \leq 6$. The only rank 2 group containing $\mathrm{U}(2)$ of dimension at most 6 is $\mathrm{SO}(4)$. If the $\mathrm{U}(2)$ action were to extend to $\mathrm{SO}(4)$, it would have to have the same orbits by the submetry lemma. But the 4-dimensional principal $\mathrm{U}(2)$ orbits have infinite fundamental group whereas a 4dimensional quotient of $\mathrm{SO}(4)$ has finite fundamental group.

Finally, since the commuting actions by $\mathrm{U}(2)$ and $\mathrm{T}^{2}$ on $\mathrm{SU}(3)$ only have id in common, the induced $\mathrm{U}(2)$ action on $E^{6}$ is effective.

REMARK. It is interesting to note that the normal homogeneous Aloff-Wallach space, $E_{1,1,-2}$, admits actions of any possible cohomogeneity $k$, with $0 \leq k \leq 7$.

4. Cohomogeneity Four. In this section we complete our discussion of isometry groups of Eschenburg spaces. The biquotient action of the maximal torus in $\mathrm{SU}(3) \times \mathrm{SU}(3)$ induces an isometric action of a 3 -torus $\mathrm{T}^{3}$ on $E_{\bar{a}, \bar{b}}$. Since any effective action by an abelian group has trivial principal isotropy group, the quotient is four dimensional, i.e. an Eschenburg space in $E_{\bar{a}, \bar{b}} \in \mathcal{E}-\mathcal{E}_{2}$ has cohomogeneity 4 . We now show that this is the connected group of isometries.

Theorem 4.1. The identity component of $\operatorname{Iso}\left(E_{\bar{a}, \bar{b}}\right)$ for any $E_{\bar{a}, \bar{b}} \in \mathcal{E}-\mathcal{E}_{2}$ is the 3-torus $\mathrm{T}^{3}$.

Proof. From the rank rigidity theorem it follows that $\operatorname{Iso}\left(E_{\bar{a}, \bar{b}}\right)$ has rank 3 , so if $\operatorname{dim} \operatorname{Iso}\left(E_{\bar{a}, \bar{b}}\right)>3$ a finite cover of it will contain $\mathrm{S}^{3} \times \mathrm{T}^{2}$. 
Suppose now that $\mathrm{G}=\mathrm{S}^{3} \times \mathrm{T}^{2}$ acts almost effectively and isometrically on $E_{\bar{a}, \bar{b}}$. One easily sees that the group $\mathrm{T}^{3} \subset \mathrm{G}$ of natural isometries has isotropy group $\mathrm{T}^{2}$ at the point $p \in E_{\bar{a}, \bar{b}}$ corresponding to the identity matrix of $\mathrm{SU}(3)$. In particular $\mathrm{G}_{p}$ has rank 2 and therefore its identity component is either $\mathrm{S}^{3} \times \mathrm{S}^{1}$ or $\mathrm{T}^{2}$. In the first case the orbit $\mathrm{G} p$ is a circle and in the second case a 3-dimensional lens space.

We rule out the first case as follows: Since $S^{3} \times S^{1}$ is a normal subgroup of G, the circle orbit $C=\mathrm{G} p$ is fixed by $\mathrm{S}^{3} \times \mathrm{S}^{1}$. First assume that the $\mathrm{S}^{1}$ factor, or some non-trivial cyclic subgroup $\Gamma$ of it, fixes a 2 -dimensional or a 4-dimensional subspace of the 6-dimensional normal space to $C$. Then the $S^{3}$ factor acts as the direct sum of a trivial and a 3 or 4 dimensional irreducible representation on this normal space since it preserves the fixed point set of $\Gamma$. This implies that the $S^{3}$ factor acts fixed point homogeneously on $E_{\bar{a}, \bar{b}}$. So by (1.6) we conclude that the $\mathrm{S}^{1}$ factor must act freely on the normal 5 -sphere, i.e., it is the Hopf action. This means that the $\mathrm{S}^{3}$ representation is a 3 -dimensional complex representation. In particular it is either fixed point homogeneous, or it is the sub-action of $\mathrm{SO}(3)$ in $\mathrm{SU}(3)$. In the latter case the action of $\mathrm{S}^{3} \times \mathrm{S}^{1}$ is effectively the tensor product action of $\mathrm{SO}(3) \times \mathrm{SO}(2)$ which has cohomogeneity one on the normal 5 -sphere. Thus the $\mathrm{S}^{3}$ action on $E_{\bar{a}, \bar{b}}$ has fixed point cohomogeneity one, contradicting Theorem (1.6).

In the second case we claim that the Eschenburg space lies in $\mathcal{E}_{2}$ : The identity component $\mathrm{T}^{2}$ of the isotropy group $\mathrm{G}_{p}$ contains a circle, $\mathrm{S}$, in the $\mathrm{T}^{2}$ factor of $\mathrm{G}$. In particular, $\mathrm{S}$ fixes the 3-dimensional orbit $\mathrm{G} p$. Since the fixed point set of $\mathrm{S}$ has even codimension, it follows again from (1.6) that the homogeneous space $\mathrm{G} p$ is a component of the fixed point set of S. Note that $\mathrm{S}$ consists of natural isometries, and one easily analyzes the fixed point sets of such isometries (see [FZ2]). The circle actions $\mathrm{S}$ and $\mathrm{S}_{\bar{a}, \bar{b}}^{1}$ define an action of a 2-torus on $\mathrm{SU}(3)$ and a fixed point of $\mathrm{S}$ on $E_{\bar{a}, \bar{b}}$ corresponds to $g \in \mathrm{SU}(3)$ whose orbit under the 2-torus is one dimensional. Hence there exist a circle action $\mathrm{S}_{\bar{c}, \bar{d}}^{1}$ inside this two torus which fixes $g$ which means that $i \bar{c}$ (considered as a diagonal matrix in $\mathfrak{s u}(3)$ ) is conjugate to $i \bar{d}$ via the matrix $g$. Thus, up to permutation, the diagonal entries agree. If the diagonal entries are distinct, $g$ must be diagonal (up to permutations of the rows), which implies that the fixed point set of $\mathrm{S}$ is a circle in $E_{\bar{a}, \bar{b}}$. Since the fixed point set is 3-dimensional, we conclude they both have a double eigenvalue which agrees. Since by assumption the component of the fixed point set also contains Id, it follows that $g$ lies in one of the three blocks $\mathrm{U}(2) \subset \mathrm{SU}(3)$.

We finally claim that the image $\mathrm{U}(2) / / \mathrm{S}_{\bar{a}, \bar{b}}^{1}$ in $E_{\bar{a}, \bar{b}}$, which agrees with $\mathrm{G} p$ by assumption, is homogeneous if and only if the induced action by $\mathrm{S}_{\bar{a}, \bar{b}}^{1}$ on $\mathrm{U}(2)$ is one sided, which is precisely when the Eschenburg space has cohomogeneity two (cf. (3.1) where $\mathrm{U}(2)$ is chosen as the 12 block). To see this, we observe that the universal cover of $\mathrm{U}(2) / / \mathrm{S}_{\bar{a}, \bar{b}}^{1}$ can be written as $\mathrm{S}^{1} \times \mathrm{S}^{3} / \mathrm{S}_{p, q}^{1}$ for some $p, q \in \mathbb{Z}$, where the $\mathrm{S}^{1}=\mathrm{S}_{p, q}^{1}$ action is given by $z \cdot(w, t)=\left(z w, z^{p} t z^{-q}\right)$. Furthermore, $\mathrm{S}^{3}$ is endowed with a left invariant, $S^{1}$-right invariant metric and $S^{1} \times S^{3}$ carries the product metric. The homogeneous orbit $\mathrm{G} p$ is, up to covering, a Berger sphere since the rank of its isometry group is 2 . This in turn implies that the natural $\mathrm{T}^{2}$ action on it has two circle orbits and the length of these two orbits coincide. On $\mathrm{U}(2) / / \mathrm{S}_{\bar{a}, \bar{b}}^{1}$ we also have a natural isometric $\mathrm{T}^{2}$ action coming from torus actions on the left and on the right. These $\mathrm{T}^{2}$ actions would have to coincide since they are both maximal tori in the full isometry group. The inverse image of the singular orbits of this $\mathrm{T}^{2}$ action in $\mathrm{S}^{1} \times \mathrm{S}^{3}$ are two dimensional tori namely $\mathrm{S}^{1} \times \mathrm{S}^{1}$ and $\mathrm{S}^{1} \times j \mathrm{~S}^{1}$ for the quaternion $j$. 
Clearly the volume of these two tori coincide and hence the length of $\left(S^{1} \times S^{1}\right) / / S^{1}$ and $\left(\mathrm{S}^{1} \times j \mathrm{~S}^{1}\right) / / \mathrm{S}^{1}$ can only be the same if the $\mathrm{S}^{1}$ actions on the two tori have orbits of the same length. But this implies that $|p-q|=|p+q|$, i.e., $p=0$ or $q=0$, and the action is one sided. $\square$

5. Fundamental Groups. It is a simple and well known fact that any finite group is the fundamental group of a non-negatively curved manifold. A basic question is if there are any obstructions in positive curvature other than finiteness of the fundamental group.

In analogy with the situation in negative curvature, where a theorem of Preismann asserts that any abelian subgroup must be cyclic, Chern proposed the same obstruction for positive curvature in [Ch]. However, in [Sh1], it was shown that two well known positively curved manifolds, the Aloff-Wallach space $A_{1,1}$ and the cohomogeneity one Eschenburg space $E_{1,1,2}$, both admit a free, isometric $\mathrm{SO}(3)$-action. In particular, any finite group $\mathrm{F} \subset \mathrm{SO}(3)$ containing $\mathbb{Z}_{2} \oplus \mathbb{Z}_{2}=\mathrm{S}(\mathrm{O}(1) \mathrm{O}(1) \mathrm{O}(1))$ is the fundamental group of a positively curved manifold contradicting Chern's conjecture. Soon after infinitely many examples with fundamental group $\mathbb{Z}_{3} \oplus \mathbb{Z}_{3}$ were found in [GSh] (one of these was also found independently in [Ba2]). These as well as other groups were in fact the first non-space form groups, to be exhibited in positive curvature. We proceed to exhibit an abundance of examples of positively curved manifolds with non-space form groups as fundamental groups.

In all cases we have encountered, we only get something interesting from subgroups of the identity component. Moreover, as observed in [GSh], if $\Gamma \subseteq \mathrm{Iso}_{0}$ acts freely, then $\Gamma$ intersects any maximal torus in a cyclic group. According to Borel [Bo] a compact, connected group $G$ has a non-toral $\mathbb{Z}_{p} \oplus \mathbb{Z}_{p}$ if and only if $\pi_{1}(\mathrm{G})$ has $p$-torsion. From our description of isometry groups here, this already suggests not to expect more interesting free, finite sub-actions from $\mathrm{U}(2) \times \mathrm{SO}(3)=\mathrm{Iso}_{0}\left(E_{p}\right)$ than from $\mathrm{SU}(2) \times \mathrm{SO}(3)$. Similarly, only when all $p_{i}$ are odd, is there a chance that $\operatorname{Iso}_{0}\left(E_{\bar{p}}\right)=\mathrm{T}^{2} \times \mathrm{SO}(3)$ has interesting free finite sub-actions, and they all come already from $\mathrm{S}^{1} \times \mathrm{SO}(3)$. Evidently, the cohomogeneity four Eschenburg spaces do not admit free, isometric actions of non-cyclic, abelian subgroups.

Since $\mathrm{SO}(3)$ has many interesting finite subgroups that are not space form groups, it is worthwhile to determine for which $E_{\bar{p}} \in \mathcal{E}_{2}$ an $\mathrm{SO}(3) \subset \operatorname{Iso}\left(E_{\bar{p}}\right)$ acts freely. Moreover, $\mathrm{SO}(3)$ is a normal subgroup in $\operatorname{Iso}\left(E_{\bar{p}}\right)$, so the isotropy groups of the action are simply its intersection with the regular and singular isotropy groups of the cohomogeneity two action. By considering the isotropy groups corresponding to the vertex points determined in (3.3), it follows that if the action is free, then $\bar{p}=(1,1,1)$ or $\bar{p}=(1,1,2)$. This recovers the main result in [Sh1] (see also [Cha] where it is shown that $A_{1,1}$ is a fat, $\mathrm{SO}(3)$-principal bundle over $\mathbb{C P}^{2}$.)

TheOREM 5.1. $\mathrm{SO}(3) \subseteq \operatorname{Iso}\left(E_{\bar{p}}\right)$ acts freely if and only if $\bar{p}=(1,1,1)$ or $\bar{p}=$ $(1,1,2)$.

Although $\mathrm{SO}(3)$ does not act freely on other Eschenburg spaces, we will see that any finite subgroup $\mathrm{F} \subset \mathrm{SO}(3)$ in fact acts freely on a large class of these spaces. As we will see, the same assertion holds for the product of many cyclic groups with $\mathrm{F} \subset \mathrm{SO}(3)$.

We begin with the cohomogeneity one spaces $E_{p}$, where $\operatorname{Iso}\left(E_{p}\right)_{0} \cong \mathrm{SO}(3) \times \mathrm{U}(2)$. For any finite group $\Gamma \subset \mathrm{SU}(2) \times \mathrm{SU}(2)$, we let $\Gamma(p) \subset \mathrm{Iso}\left(E_{p}\right)$ be the image. Recall that $\mathrm{H}=\left\{( \pm \mathrm{id})^{p+1},( \pm \mathrm{id})^{p}\right\}$ is the kernel of the action. 
Proposition 5.2. For a finite group $\Gamma \subset \mathrm{SU}(2) \times \mathrm{SU}(2)$, the group $\Gamma(p)$ acts freely on $E_{p}$ if and only if for all $\left(\gamma_{1}, \gamma_{2}\right) \in \Gamma-\mathrm{H}$ we have the conditions

- $\left|\gamma_{1}\right| \neq\left|\gamma_{2}\right|$, and $\left(\gamma_{1}, \gamma_{2}\right) \neq \pm(-\mathrm{id}, \mathrm{id})$, and

- $\left|\gamma_{1}\right|$ does not divide $p\left|\gamma_{2}\right|$ or $\left|\gamma_{2}\right|$ does not divide $(p+1)\left|\gamma_{1}\right|$, where $\left|\gamma_{i}\right|$ denotes the order of $\gamma_{i}$.

Proof. Any element of $\mathrm{SU}(2)$ has eigenvalues $\{\lambda, \bar{\lambda}\}$ for some $\lambda \in \mathrm{U}(1)$; let $\gamma_{i}$ have eigenvalues $\left\{\lambda_{i}, \bar{\lambda}_{i}\right\}$. Clearly $\Gamma(p)$ fails to act freely only if for some $\left(\gamma_{1}, \gamma_{2}\right) \in$ $\Gamma-\mathrm{H}$ there is a $\left(\operatorname{diag}\left(z, z, z^{p}\right), \operatorname{diag}\left(1,1, z^{p+2}\right) \in \mathrm{S}_{p}^{1}\right.$ such that $\operatorname{diag}\left(z, z, z^{p}\right) \gamma_{1}$ and $\operatorname{diag}\left(1,1, z^{p+2}\right) \gamma_{2}$ are conjugate, i.e., have the same set of eigenvalues. So the action is free unless the sets $\left\{z \lambda_{1}, z \bar{\lambda}_{1}, z^{p}\right\}$ and $\left\{\lambda_{2}, \bar{\lambda}_{2}, z^{p+2}\right\}$ are the same. This happens only if either: (a) $\lambda_{1}= \pm \lambda_{2}$, or $\lambda_{1}= \pm \bar{\lambda}_{2}$ or (b) $\lambda_{1}^{p}=\lambda_{2}^{p+1}$ or $\lambda_{1}^{p}=\bar{\lambda}_{2}^{p+1}$. Case (a) corresponds to $\left|\gamma_{1}\right|=\left|\gamma_{2}\right|$ or $\left(\gamma_{1}, \gamma_{2}\right)= \pm\left(-\mathrm{id}\right.$, id) while in case (b) we have that $\left|\gamma_{2}\right|$ divides $\left|\gamma_{1}\right|(p+1)$ and $\left|\gamma_{1}\right|$ divides $\left|\gamma_{2}\right| p$.

This has some interesting consequences. For instance, if $\Gamma \subset \mathrm{SU}(2) \times\{\mathrm{id}\}$, and $p$ is even, then $\Gamma(p)$ acts freely if none of the orders of elements of $\Gamma \backslash\{-$ id $\}$ divide p. Also, $\Gamma(p) \times \mathbb{Z}_{q}$ acts freely on $E_{p}, p$ even if $\operatorname{gcd}(|\Gamma|, q)=\operatorname{gcd}(|\Gamma|, p)=1$ or $\operatorname{gcd}(|\Gamma|, q)=\operatorname{gcd}(|\Gamma|, p+1)=1$ We deal with $\Gamma(p)$ and $\mathbb{Z}_{q} \times \Gamma(p)$ for $\Gamma \subset\{\operatorname{id}\} \times \operatorname{SU}(2)$, and $p$ is odd similarly. This proves the cohomogeneity one part of Theorem B in the introduction. Note that the quaternion group $\Gamma=\{ \pm 1, \pm i, \pm j, \pm k\} \subset \mathrm{SU}(2)$ corresponds to $\Gamma(p)=\mathbb{Z}_{2} \oplus \mathbb{Z}_{2} \subset \mathrm{SO}(3)$, and that $\mathbb{Z}_{q} \times\left(\mathbb{Z}_{2} \oplus \mathbb{Z}_{2}\right)=\mathbb{Z}_{2 q} \oplus \mathbb{Z}_{2}$.

For the cohomogeneity two Eschenburg spaces $E_{p_{1}, p_{2}, p_{3}}$ with all $p_{i}$ odd, recall that the right action by $\mathrm{SU}(2)$ acts effectively as $\mathrm{SO}(3)$. For these we have

Proposition 5.3. Any finite group $\mathrm{F} \subset \mathrm{SO}(3)$ acts freely on an infinite family of spaces $\mathrm{E}_{\bar{p}} \in \mathcal{E}_{2}$, in particular when all $p_{i}$ are odd and distinct and $p_{i} \equiv 1 \bmod |\mathrm{F}|$.

Proof. As usual we let $\mathrm{F}^{*}$ be the inverse image of $\mathrm{F}$ by the map $\mathrm{SU}(2) \rightarrow \mathrm{SO}(3)=$ $\mathrm{SU}(2) /\{ \pm \mathrm{id}\}$. In particular, the order of any element of $\mathrm{F}^{*}$ divides the order of $\mathrm{F}$.

Let $p_{1}, p_{2}, p_{3}$ be three distinct primes, all congruent to 1 modulo $|\mathrm{F}|$ (infinitely many such primes exist by Dirichlet's theorem on primes in arithmetic progression). As in the proof above we see that the action of $\mathrm{F}$ on the space $E_{p_{1}, p_{2}, p_{3}}$ fails to be free only if the sets $\left\{z^{p_{1}}, z^{p_{2}}, z^{p_{3}}\right\}$ and $\left\{\gamma, \bar{\gamma}, z^{\sum p_{i}}\right\}$ are the same for some $z \in \mathrm{U}(1)$. Without loss of generality we may assume that $z^{p_{1}}=\gamma, z^{p_{2}}=\bar{\gamma}, z^{p_{1}+p_{2}}=1$. Since in particular $p_{1}$ and $p_{2}$ are relatively prime, we can find integers $a, b$ such that $a p_{1}+b p_{2}=$ 1. Then $z=z^{a p_{1}+b p_{2}}=\gamma^{a-b}$. But since also $p_{1}$ and $p_{2}$ are both congruent to 1 modulo $|\mathrm{F}|$, we have: $\gamma=z^{p_{1}}=\gamma^{(a-b) p_{1}}=\gamma^{a-b}$ and $\bar{\gamma}=z^{p_{2}}=\gamma^{(a-b) p_{2}}=\gamma^{a-b}$. This implies $\gamma=\bar{\gamma}= \pm$ id which is simply the kernel of the action. Hence, $\mathrm{F}^{*} /\{ \pm \mathrm{id}\}=\mathrm{F}$ acts freely and isometrically on each of these spaces.

\section{REFERENCES}

[AW] S. Aloff and N. Wallach, An infinite family of distinct 7-manifolds admitting positively curved Riemannian structures, Bull. Amer. Math. Soc., 81 (1975), pp. 93-97.

[Ba1] Y. BAZAIKIN, On a family of 13-dimensional closed Riemannian manifolds of positive curvature, (Russian) Sibirsk. Mat. Zh., 37 (1996), pp. 1219-1237; translation in Siberian Math. J., 37 (1996), pp. 1068-1085.

[Ba2] Y. BAZAikin, A manifold with positive sectional curvature and fundamental group $\mathbb{Z}_{3} \oplus \mathbb{Z}_{3}$, Siberian Math. J., 40 (1999), pp. 834-836.

[BB] L. BÉRARD BERGERY, Quelques exemples des variétés riemanniennes complètes non compactes à courbure de Ricci positive, C. R. Acad. Sci. Paris, 302 (1986), pp. 159-161. 
[Be] M. BERGER, Les variétés riemanniennes homogènes normales simplement connexes à courbure strictement positive, Ann. Scuola Norm. Sup. Pisa, 15 (1961), pp. 179-246.

[Bo] A. Borel, Sous-groupes commutatifs et torsion des groupes de Lie compacts connexés, Tohuku Math. J., 3 (1961), pp. 216-240.

[Ch] Problem Section in Proc. of the US-Japan Seminar in Differential Geometry, Kyoto, Japan (1966).

[Cha] L.M. Chaves, A theorem of finiteness for fat bundles, Topology, 33 (1994), pp. 493-497.

[CEZ] C. Escher, T. Chinburg And W. Ziller, Topological properties of Eschenburg spaces and 3-Sasakian manifolds, Math. Ann., to appear.

[Es1] J.-H. Eschenburg, New examples of manifolds with strictly positive curvature, Invent. Math., 66 (1982), pp. 469-480.

[Es2] J.-H. Eschenburg, Inhomogeneous spaces of positive curvature, Diff.Geom.Appl., 2 (1992), pp. $123-132$.

[Es3] J.-H. EschenburG, Freie isometrische Aktionen auf kompakten Lie Gruppen mit positiv gekrümmten Orbiträumen, Habilitation, Uni Münster Math. Institut, 1984.

[FZ1] L. A. Florit AND W. Ziller, On the topology of positively curved Bazaikin spaces, Preprint 2006.

[FZ2] L. A. Florit ANd W. Ziller, Orbifold fibrations of Eschenburg spaces, Preprint 2006.

[GK] K. Grove And C. W. KIM, Positively curved manifolds with low fixed point cohomogeneity, J. Differential Geom., 67 (2004), pp. 1-33.

[GS1] K. Grove And C. Searle, Positively curved manifolds with maximal symmetry rank, J. Pure Appl. Algebra, 91 (1-3) (1994), pp. 137-142.

[GS2] K. GRove AND C. SEARLe, Differential topological restrictions by curvature and symmetry, J. Differential Geom., 47 (1997), pp. 530-559.

[GSh] K. Grove And K. Shankar, Rank two fundamental groups of positively curved manifolds, J. Geometric Anal., 10 (2000), pp. 679-682.

[GWZ] K. Grove, B. Wilking and W. Ziller, Positively curved cohomogeneity one manifolds and 3-Sasakian geometry, preprint.

$[\mathrm{KN}] \quad$ S. Kobayashi and K. Nomizu, Foundations of differential geometry, Volume II, John Wiley Interscience, 1969.

[KS] M. KRECK AND S. Stolz, Some non diffeomorphic homeomorphic homogeneous 7 manifolds with positive sectional curvature, J. Diff. Geom., 33 (1991), pp. 465-486.

[Kr1] B. Kruggel, A homotopy classification of certain 7-manifolds, Trans. Amer. Math. Soc., 349 (1997), pp. 2827-2843.

[Kr2] B. Kruggel, Homeomorphism and diffeomorphism classification of Eschenburg spaces, Quart. J. Math. Oxford Ser. (2), 56 (2005), pp. 553-577.

[On] A. L. ONISHCHIK, Inclusion relations between transitive compact transformation groups, Trudy Moskov. Math. Obsc., 11 (1962), pp. 199-242. English translation in Amer. Math. Soc. Transl., 50 (1996), pp. 5-58.

[Se] C. SeArle, Cohomogeneity and positive curvature in low dimensions, Math. Z., 214 (1993), pp. 491-498.

[Sh1] K. ShankAR, On the fundamental groups of positively curved manifolds, J. Differential Geom., 49 (1998), pp. 179-182.

[Sh2] K. SHANKAR, Isometry groups of homogeneous spaces with positive sectional curvature, Diff. Geom. \& Appl., 14 (2001), pp. 57-78.

[Sh3] K. Shankar, Strong inhomogeneity of Eschenburg spaces (with an Appendix by M. Dickinson and K. Shankar), Mich. Math. J., 50 (2002), pp. 125-142.

[Ta] I. A. TAImanov, On totally geodesic embeddings of 7-dimensional manifolds in 13dimensional manifolds of positive sectional curvature, Sib. Math., 187 (1996), pp. 18531867.

[Wa] N. Wallach, Compact homogeneous Riemannian manifolds with strictly positive curvature, Ann. of Math., 96 (1972), pp. 277-295.

[WZ] M. WANG AND W. ZILler, On isotropy irreducible Riemannian manifolds, Acta Math., 167 (1991), pp. 223-261.

[Wi1] B. WILKING, The normal homogeneous space $(\mathrm{SU}(3) \times \mathrm{SO}(3)) / \mathrm{U}^{*}(2)$ has positive sectional curvature, Proc. Amer. Math. Soc., 127 (1999), pp. 1191-1194.

[Wi2] B. Wilking, Torus actions on manifolds of positive sectional curvature, Acta Math., 191 (2003), pp. 259-297.

[Wi3] B. Wilking, Positively curved manifolds with symmetry, Ann. of Math., 163 (2006), pp. 607-668.

[Zi] W. ZILLER, Homogeneous spaces, biquotients, and manifolds with positive curvature, Lecture Notes 1998, unpublished. 
